\title{
The Research and Implementation about AFIS
}

\author{
Yuan Renmin \\ Marine college Shandong jiaotong university, China \\ e-mail: yrm0019@163.com
}

Keywords: fingerprint identification; Component technology; IC card applications.

\begin{abstract}
Automatic identification technology becomes a urgent need of production and life, authentication technology gained worldwide attention because of its high reliability, fingerprint identification technology which applied to social security system can accurately determine protects a person's identity and prevent the phenomenon of the pension of falsely claim that solves this one long-term puzzling problem. Based on the preeminent fingerprint identification algorithm is improved, and combining with actual demand the improved algorithm, Using the fingerprint identification and IC card combination way realization of distributed fingerprint authentication system collection. Through the practical efficiency test analysis proves the whole system is feasible, and the running effect is good by practical application.
\end{abstract}

\section{Preface}

Due to human body features with no duplicated characteristics, people put eyes turned to biometrics. Computer fingerprint identification automatic identification technology is the earliest and the most practical and the mature technology among the identification technology. through the detailed analysis for automatic fingerprint identification system needs, and according to the system's demand for fingerprint identification system for the overall analysis and design, first fingerprint collection and processing scheme design and implementation, and then the fingerprint information processing to carry on the detailed planning, and finally puts forward some fingerprint identification authentication center of the whole system scheme.

As computer processing fingerprint, fingerprint only involve some limited information, but not exact match than on the algorithm, the results can not guarantee $100 \%$ accurate. Fingerprint identification system to measure the specific application of an important symbol is the recognition rate [5]. Mainly consists of two parts, refuse to sub-rate (FRR) and false positive rate (FAR). We can adjust according to different uses of these two values. FRR and FAR is inversely proportional. With 0 1.0 or expressed as a percentage of this number. ROC (Receiver Operating Curve) - curve shows the relationship between FAR and FRR. Figure 1

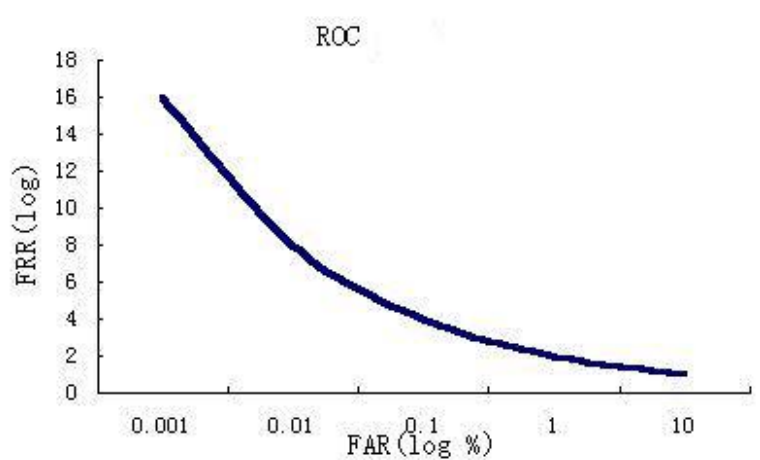

Figure 1 Receiver Operating Curve

Although the reliability of fingerprint identification system exists, but its safety level of reliability than the same "user ID + password" program is much more safe. For example, four-digit password using the system [7], the probability is $0.01 \%$ unsafe, if the error rate of $0.01 \%$ with the use of fingerprint identification systems, as a dishonest person can be a period of time trying all possible passwords Therefore, the password is not secure four, but he is absolutely impossible for him to find a thousand people go to all the fingers (ten fingers) are tested again. Because of this, authorities believe, in the application of $1 \%$ false positive rate is acceptable. 


\section{The Principle of Fingerprint Identification}

Automatic fingerprint Identification system work mode can be divided into two types: Verification mode and Identification mode. Verification mode also called one-to-one ratio (l: 1), principle for Matching: fingerprint pre-register to sample database and set a logo yards, when matching, inputting fingerprint and input identification code, system identification code from database according to extracting fingerprint sample and own fingerprint compares. Identification mode which also called a pair of dobby (l: N Matching), is to treat entry fingerprints and sample database of all fingerprints were compared, until find one match fingerprints or search the entire sample database are no corresponding fingerprints after the conclusion.

Automatic fingerprint identification system mainly involves image collection, pretreatment, feature extraction, and feature matching and so on several parts. The system consists of image input, pretreatment, feature extraction, classification and matching parts, among these five parts pretreatment part can divide again for image segmentation, image enhancement, binary conversion and refining etc.

\section{The Fingerprint Identification Algorithms.}

Fingerprint identification include the following steps: fingerprint image pretreatment, fingerprint characteristic extraction, fingerprint characteristic comparison, a typical fingerprint identification algorithm process.

The algorithm for the fingerprint of image processing uses the many kinds of optimization algorithm, including the frequency domain and time domain two ways of combining the filtering processing technology which be able to effectively filter fingerprint noise. At the same time its unique fingerprint quality (such as dry humidity) evaluation factors, can effective adjustment the quality of pixels of normal direction. For dryness and broken mark fingerprints, by analyzing the fingerprint direction can effectively compensate amount. Total processing steps can reach 17 steps above. The image processing of simplified as figure 2 shows:

Original figure after image enhancement after

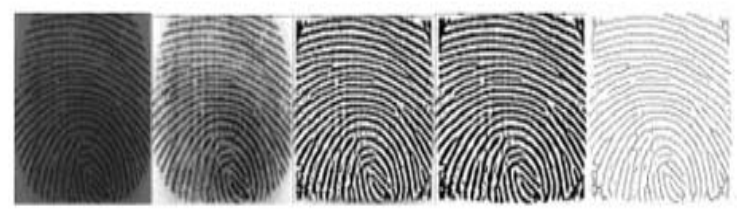

Figure 2 simplified diagram of image processing process smooth processing after binaryzation after thinning

\section{Analysis and Design of The Fingerprint Identification System}

Fingerprint identification authentication center system composes of the database part, system management section, application server part, user management workstations and business management workstation five major components. Attend insurance personnel, system administrators, business personnel, information of attend insurance personnel are the most basic object class, the main operations are attend insurance personnel information registration, fingerprint acquisition, fingerprint verification, validation information query. The most main activity is the fingerprint collection and fingerprint verification.

Social security card information collection service system strives to construct an electronic and effective, synthetic multi-function graphic information management and survival verification system, annuities receivers survive verification validation are judged through fingerprint identification technology as the main technical means, supplemented by other graphic information (signature, photo, ID, bank account passbook, etc.) verification methods, which makes the social security organization endowment insurance business system more perfect. Social security card information collection service system includes the following function module: attend insurance personnel file, the social security core platform. The core platform is the core part of the whole social insurance system, which including endowment annuity, unemployment insurance, medical insurance, insurance against injury at work and birth insurance by business divisions and each 
subsystem are both run separately or combined together operation arbitrarily. Data sources of social insurance management information system include attend insurance unit, bank, taxation, designated medical establishment retail medicamentarius information system, the core platform has also data interface with these external system, so that handing data exchange.

\section{Implementation of Fingerprint Identification System}

The fingerprint identification system is designed in strict accordance with the labor bureau work requirements and safety guidelines, generality, portability, more information and higher safety requirements of the system are full consideration. The fingerprint collection and fingerprint verification are the most main activity.

A.implementation of fingerprint acquisition Maintaining the Integrity of the Specifications

Fingerprint acquisition subsystem in the program uses PowerBuilder compile which makes the fingerprint instrument operation and fingerprint identification algorithm encapsulation into DLL and proceeds necessary function declaration.

B. implementation of fingerprint authentication

Fingerprint identification authentication subsystem makes fingerprint identification algorithm encapsulation into DLL using PowerBuilder compile and in PowerBuilder in necessary function declarations, then according to the following processes realize fingerprint identity authentication, fig. 3 shows.

Note: When the certification by pressing the fingers, if the user account authentication window has information, then this action as a verification for this user, or as identification all users.

The fingerprint authentication platform provides a complete biometric authentication features: safety performance, wide range of applications, no platform, support multiple architectures (single, C / S, B / S, N-Tier) for seamless integration, support for all development languages. Especially in the Internet browser with a variety of Script Language (VBScript, JavaScript) and ActiveX technology, using a standard Web browser to achieve the original client software required to achieve a particular strong authentication function, a large number of saving development costs.

\section{Implementation of IC Card Data Access}

The system used MD5 (Message-Digest algorithm 5) one-way encryption algorithm, which consisted of MD2, MD3, MD4 developed from a one-way function algorithm (that was, HASH algorithm), which was an internationally renowned public-key encryption algorithm standard RSA, which developed by the first designer R.Rivest in the early 90s of last century. The most important function of MD5, the large files of different formatted of information in the software used to sign digital signature private key before the "compressed" into a confidential format, the key is that this "compression" is not reversible. For MD5, there are two characteristics are very important, first, any two plaintext data, cipher text after encryption can not be the same, the second is a clear text any data after the encrypted, the result must always be unchanged. IC card reader on the general process has been improved to achieve the same procedure used in the preparation of PowerBuilder, Dynamic Link Library by calling the IC card reading and writing.

\section{Conclusion}

Fingerprint authentication system applied to the social endowment insurance system, has basically met the needs of the first set, can be achieved, or for retirees who retired fingerprint registration formalities, and then retired regular or irregular acquisition of the original fingerprint to compare the fingerprint to verify the retirees to the survival of the state's purposes. Fingerprint Identification System fingerprint authentication state and status of real-time feedback to the business system. The pensions system in the real alternative to the traditional manual identification of the identity of the insured's work, improve efficiency, effectively prevent the occurrence impersonator pensions, more easily verify the conditions of the late management. 


\section{References}

[1] Tianjie,Yang xin. Theory and application of Biometrics identification technology. Publishing House of Electronics Industry..2006:56-77

[2] Wang shengguo. Fingerprint recognition algorithm research and realization implement. China excellent master degree theses full-text database. Hainan normal university. 2007:38 41

[3] Ren chunxiao. Fingerprint algorithm study platform design development and application. Shandong university. 2007:66 79

[4] Liang chuan. The Scenario and Application of Fingerprint Authentication with Electronic Request-Authorization System in Traffic Business. Sichuan university. 2007,(4)

[5]A. Cavoukian, J. Stoianov. A Positive-Sum Technology that Achieves Strong. Authentication, Security and Privac. 2007,(3)

[6]D. Simon, Z. Jortega, S. Llanas and J. Gonzalez. Minutiae Extractios chemefor fingerprint recognition systems. International Conferenceon Image Processing(ICIP), Greece, 2001,10:7 10

[7]W. Green, D. John. Internet and Distributed Application Development Beijing mechanical industry press.. 2004(7)

[8] K. J. Anil, P. Bolle. An Identity-Authentication System Using Fingerprints Proceedings of The IEEE. 1997,9(9):85

[9] A. Bishmu, S. Das, and S. Nandy. An improved Algorithm for Point Set Pattern Matehing under Rigid Motion.CIAC,LNCS 2653. 2003:36 45 